\title{
Set up and characterization of a measuring system for PQ measurements in a MV site with PV generation
}

\author{
Gabriella Crotti, Domenico Giordano \\ Istituto Nazionale di Ricerca Metrologica, Strada delle Cacce, 91, 10135 Torino, Italy
}

ABSTRACT

The features of a Power Quality measurement system which includes both voltage and current transducers and a self-developed measuring instrument are described. The system is intended for measurements in substations at medium voltage level. A Rogowski coil and a voltage divider are the used transducers, whereas the measuring instrument is based on a Reconfigurable I/O-FPGA system with embedded software. Attention is focused on the procedures adopted for the characterization of the voltage and current sensors, which are carried out taking into account the expected on-site measurement conditions. An example of use of the systems for measurement of some PQ parameters in a private substation which connects an industrial load and two photo-voltaic generation plants to the public MV voltage network is presented, together with the associated voltage and current uncertainty budget. By making use of the results obtained in the characterization phase, the current and voltage measurement uncertainty can be reduced.

\section{Section: RESEARCH PAPER}

Keywords: Non-conventional transformers, PQ measurements, MV substation, PV plants

Citation: Gabriella Crotti, Domenico Giordano, Set up and characterization of a measuring system for PQ measurements in a MV site with PV generation, Acta IMEKO, vol. 4, no. 1, article 15, February 2015, identifier: IMEKO-ACTA-04 (2015)-01-15

Editor: Paolo Carbone, University of Perugia

Received January $15^{\text {st }}, 2014$; In final form November 21 ${ }^{\text {st }}, 2014$; Published February 2015

Copyright: (C) 2014 IMEKO. This is an open-access article distributed under the terms of the Creative Commons Attribution 3.0 License, which permits unrestricted use, distribution, and reproduction in any medium, provided the original author and source are credited

Funding: The research leading to the results here described is part of the European Metrology Research Program (EMRP), which is jointly funded by the EMRP participating countries within EURAMET and the European union.

Corresponding author: Domenico Giordano, e-mail: d.giordano@inrim.it

\section{INTRODUCTION}

The measurement of the quality of the electrical supply is today gaining more and more attention. This is due to the need of accurate evaluation of the power quality for the assessment of the compliance to the limits indicated for public distribution networks, the identification and location of disturbances and the verification of the impact of newly connected distributed sources [1]-[5].

As to the power quality (PQ) measurement systems, attention is generally mainly focused on the performances of the measuring instruments. Test procedures, expected performances and uncertainties are extensively prescribed for the measuring instruments only in the relevant standards [6], [7]. When dealing with PQ measurements in medium and low voltage grids, a reduction of current and voltage level is needed. To this end, the instrument transformers already available in the measurement site are generally used. However, their use for the measurement of
PQ events cannot be considered completely satisfactory and their contribution to the overall uncertainty can be significant [8]. As an alternative, non-conventional transformers are more and more considered, because of their better performances regarding linearity and frequency response and their low level power output [9]-[13]. Prescriptions for the measurement uncertainties associated to transducers used in PQ measurements are given in [15]. According to this, the percentage error has to be within $1 \%$ of the measured value for the first and second harmonic and within $5 \%$ for the higher order ones. With reference to the required performances, the features of different types of current and voltage sensors are analysed in [8]. To estimate and reduce the uncertainty of the $P Q$ parameters, the characteristics of the adopted transducers and their performance in relation to the expected measurement conditions including the circuit sensors arrangement and the surrounding environment should be known. To this 
end, an extended and focused transducer characterisation is needed.

The paper deals with a system for PQ measurement at medium voltage (MV) up to $24 \mathrm{kV}$ rms, which makes use of a voltage divider and three Rogowski coils (RC) and is completed by a measuring instrument based on a National Instrument Compact Reconfigurable I/O programmable automatic controller platform. The system is intended for indoor use in a MV site with Photo-Voltaic (PV) generation. The use of a clamp-on and flexible coil for the current measurement enables its positioning in harsh and narrow sites, where measurement conditions quite far from the laboratory ones are met, without need of power circuit disconnection and/or outage. However, the same features, flexibility and presence of a coil turn gap, make the sensor more sensitive to perturbing effects, such as those due to the position of the primary or other current-carrying conductors, and less accurate with respect to rigid, closed coils, with expected measurement uncertainty of the order of one percent or higher [17]. As to the voltage transducer, a solid insulated voltage divider is selected, to exploit its linearity and frequency performances. For this kind of transducer, the effects due to the divider capacitive coupling with close metallic elements at ground or floating potential and the environmental conditions have to be considered and controlled.

Attention is therefore focused on the characterisation procedure of the sensors that, if carefully performed under conditions that reproduce the experimental ones, can allow the decrease of the on-site measurement uncertainty and enables their use in compliance with the given accuracy limits [8], [15]. Application of the system to PQ measurements in a private $\mathrm{MV} / \mathrm{LV}$ substation that supplies an industrial load and is connected both to the public supply network and to photo-voltaic (PV) generation plants is eventually shown and discussed.

\section{THE MEASUREMENT SYSTEM}

The scheme of the PQ measurement system is shown in Figure 1. The system includes a voltage divider and three commercial flexible Rogowski coil (RC) with the associated integrator as transducers, a NI Compact Reconfigurable $\mathrm{I} / \mathrm{O}$ based measuring instrument and the connection to a GPS receiver for time synchronisation [16].

In the following, a description is given of the measuring system components and of the relevant characterisation



Figure 1. PQ measuring system scheme. tests carried out to estimate the uncertainty contributions to be considered in the on-site use of the current and voltage sensors.

\subsection{The current transducer}

The on-site operating conditions are generally quite different from those taking place in the laboratory when calibrating the system components. They can significantly affect the performances of the sensor as a function of its construction characteristics. In particular for clamp-on $\mathrm{RCs}$, the path of the primary and return conductors, the presence of external magnetic field sources and the coil nonorthogonality with respect to the primary conductor can lead to a considerable variation of the coil mutual inductance, whose amount depends on the sensor characteristics and construction solutions [18].

Taking into account the expected measurement location (measurement in a MV/LV substation on the MV side) and the range and type of currents to be measured (from hundreds of milliamperes to several tens of amperes), commercial flexible and clamp-on shielded Rogowski coils, equipped with a three channel integration system, have been selected. The rated characteristic of the RCs and associate integrator are summarised in Table 1.

To investigate the sensitivity of the used coil to influencing and perturbing factors and predict their effect on the expected measurement accuracy, an extensive characterisation of the $\mathrm{RC}$ with the associated integrator is carried out in the laboratory, by reproducing configurations similar to those expected on-site.

Characterisation of the current sensor includes:

i) calibration at power frequency and determination of the RC transformation ratio;

ii) linearity over the expected measurement range;

iii) frequency response determination;

iv) evaluation of return conductor proximity effects and perturbing source presence;

v) estimation of the temperature effect.

A scheme of the supply and measuring circuit for the calibration at power frequency and the linearity tests is shown in Figure 2a.

The current is generated by a Clarke-Hess 8100 transconductance amplifier $(0.2 \mathrm{~mA}$ to $100 \mathrm{~A}, \mathrm{DC}$ to 100 $\mathrm{kHz}$ ) supplied by a stable waveform generator (Fluke 5500). The current value is obtained by measuring the voltage

Table 1-RC coil and integrator rated characteristics.

\begin{tabular}{l|l}
\hline Type & Rogowski coil with integrator \\
\hline RC length & $\begin{array}{l}400 \mathrm{~mm} \\
44 \mathrm{~mm}^{2}\end{array}$ \\
RC section & $\begin{array}{l}20 \mathrm{~A} / \mathrm{V}, 100 \mathrm{~A} / \mathrm{V}, \ldots 10 \mathrm{kA} / \mathrm{V} \\
\text { (overrange } 1000 \%)\end{array}$ \\
Integrator selectable output ranges & $<0.01 \% /{ }^{\circ} \mathrm{C}$ \\
Integrator temperature coefficient & $0.04 \% /{ }^{\circ} \mathrm{C}$ \\
RC+integrator temperature coefficient & $1 \mathrm{~Hz}$ to $100 \mathrm{kHz}$ \\
Bandwidth of the RC+integrator & $1 \%$ \\
RC + integrator rated accuracy &
\end{tabular}


across a calibrated non-inductive shunt (Tinsley $0.05 \Omega$ standard resistor). The reference and RC signals have been acquired by two synchronised Agilent 3458A multimeters, exploiting their digitising features, with a sampling frequency varying from $1 \mathrm{kHz}$ to $1 \mathrm{MHz}$ depending on the supply frequency.

The measurements are carried out by centring the coil around the primary conductor by a rigid, but easily moveable non-conductive support to limit the uncertainty contribution due to coil positioning. Both the shape of the primary conductor and its diameter are chosen close to those of the MV cables (Figure 2b).

Linearity is investigated at $50 \mathrm{~Hz}$ by generating currents in the range $0.1 \mathrm{~A}$ to $100 \mathrm{~A}$ with the return conductor 30 $\mathrm{mm}$ distant from the coil loop and opposite with respect to the coil gap. The characterization is carried out for the range $20 \mathrm{~A} / 1 \mathrm{~V}$, exploiting the overrange capabilities of the selected coils (Table I). The relative deviations of the measured RC ratio from the rated one are found within $6 \cdot 10^{-4}$ over the investigated current range. A systematic increase of $+1 \times 10^{-3}$ is measured when the acquisition and measurement system is included in the measurement chain. A relative decrease of about $2 \times 10^{-4} \mathrm{~A}$ for currents lower than $1 \mathrm{~A}$ occurs due to the lower values of the digitiser input voltage.

Regarding the frequency investigation, the impedance magnitude and phase of the coil, without the integrator, have been first measured by an Agilent 4294A impedance analyser. Figure 3 compares the measured behaviour with that computed by considering a simple RCL coil model,
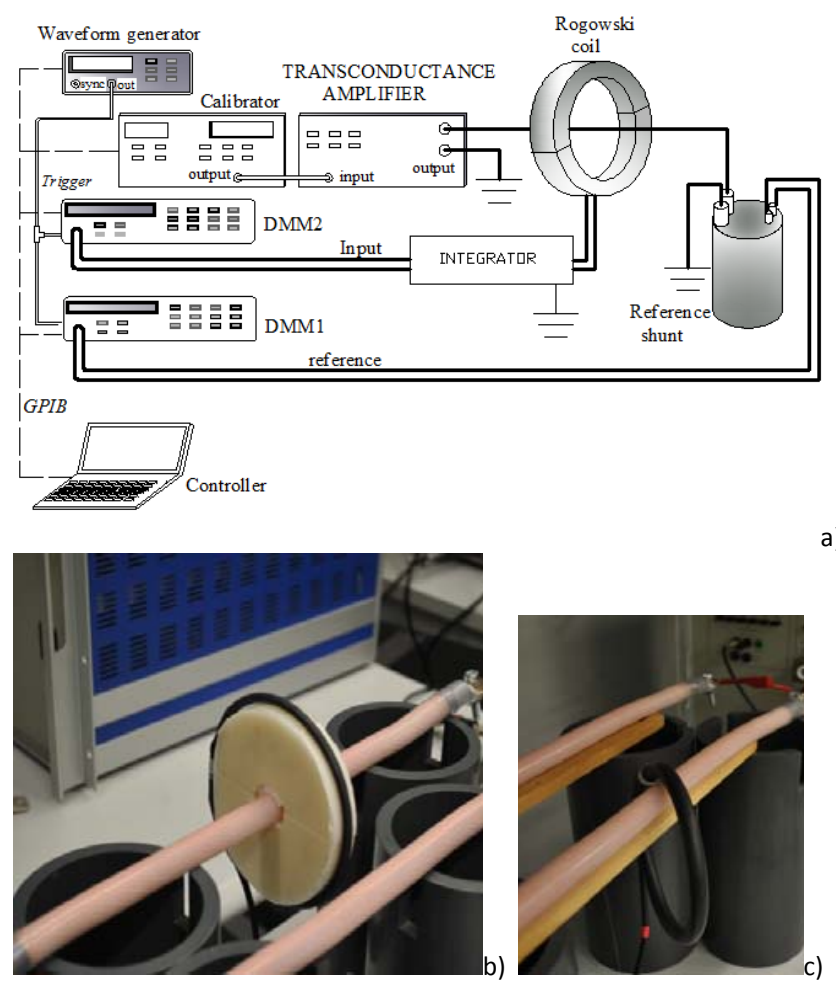

Figure 2. a) Linearity, proximity and frequency test circuit; b) RC coil experimental arrangement with the coil centered on the primary conductor; c) RC coil experimental arrangement with the coil hung from the primary conductor. whose parameter values are indicated in the graph. The resonance frequency is around $300 \mathrm{kHz}$.

The frequency behaviour of the $\mathrm{RC}$ with integrator and connection cable is then measured in the same circuit used for the linearity evaluation. Current is measured in this case by a Guildline 7320 AC shunt, whose AC/DC difference is within $200 \mathrm{ppm}$ at $100 \mathrm{kHz}$. Exploiting the use of stable and repetitive signals, evaluation of the $\mathrm{RC}$ error is carried out with the Agilent digitisers configured in DCV mode [14] from few hertz up to hundreds of hertz and in Time Equivalent Sampling mode (also named SubSampling mode) [14] for higher frequencies. Since first experimentation of the system is planned on a site where a wide harmonic spectrum can be expected (PV generation, with inverter switching frequency around $20 \mathrm{kHz}$ ), measurements are carried out up to some tens of kilohertz. Figures $5 \mathrm{a}$ and $5 \mathrm{~b}$ show the RC transformation ratio, normalised to the rated one, and the phase error measured over the range $20 \mathrm{~Hz} \div$ $40 \mathrm{kHz}$ with the return conductor $35 \mathrm{~mm}$ distant. Limit values given in [15] for the $1^{\text {st }}$ to the $50^{\text {th }}$ harmonics for the ratio and phase errors are shown for comparison by the red continuous lines. The dashed lines are limit values extrapolated to the lower and higher harmonic ranges, where no indication is presently given.

Measurements are then repeated by varying the minimum distance $d$ of the return conductor from the coil loop from $30 \mathrm{~mm}$ to $200 \mathrm{~mm}$. RC ratios normalised to the rated value are shown in Figure 6, for two distances $d$ of the return conductor (conf. i) $30 \mathrm{~mm}$ and ii) $200 \mathrm{~mm}$ ) with the coil gap external to the current loop as shown in Figure $2 b$. The behaviour measured with the gap internal, when distance $d$ is $200 \mathrm{~mm}$ (conf. iii), is also shown. A quite constant deviation of about $3.2 \times 10^{-3}$ is found between conf. i) and conf. iii).

The influence of external magnetic field sources is then investigated by measuring the coil stray signal induced by a 400 A current flowing in a conductor external to the coil loop, placed at increasing distances $D$. The induced stray signal is $2 \mathrm{mV}$ for $D=100 \mathrm{~mm}$ and reduces to $0.5 \mathrm{mV}$ when $D$ is $600 \mathrm{~mm}$.

Finally the response of the coil in the worst positioning conditions with respect to the primary conductor, that is

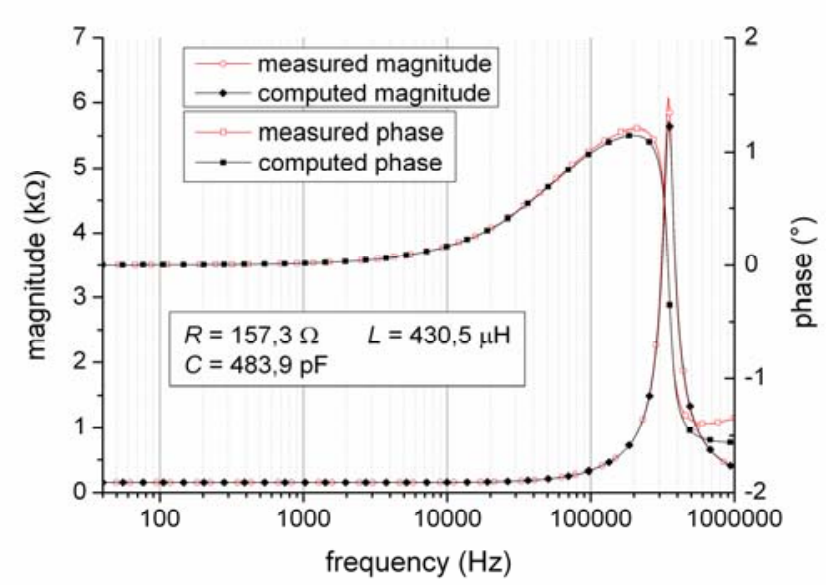

Figure 3. Measured magnitude and phase of the RC impedance. 
with the coil directly suspended on it as shown in Figure $2 \mathrm{c}$, is also determined. The relative deviation of the RC ratio from the value measured with the centred coil increases up to $2 \%$, when the gap is close to the conductor.

\subsection{The voltage transducer}

The voltage sensor is a resistive divider with rated scale factor SF 10000, $1 \mathrm{M} \Omega$ rated load, rated primary voltage $U_{\mathrm{m}}=24 \mathrm{kV}$, withstand voltage $50 \mathrm{kV}$, rated accuracy class 0.5 . The divider is polyurethanic resin insulated and is equipped with a $10 \mathrm{~m}$ coaxial cable.

Dielectric tests (power frequency withstand voltage, impulse test voltage, partial discharge test) are first carried out and successfully passed by the divider.

Characterisation tests performed include:

i) $\mathrm{SF}$ and phase errors determination at $50 \mathrm{~Hz}$ up to $24 \mathrm{kV} / \sqrt{3}$;

ii) proximity effects analysis at $50 \mathrm{~Hz}$;

iii) frequency response;

iv) temperature dependence.

The divider $\mathrm{SF}$ is measured at $50 \mathrm{~Hz}$ by comparison with an INRIM standard measurement voltage transformer (uncertainty $50 \mathrm{ppm}$ and $50 \mu \mathrm{rad}$ for their ratio and phase errors respectively). From $5 \mathrm{kV}$ to $12 \mathrm{kV}$ a relative decrease of $1 \cdot 10^{-3}$ of the SF value and an increase in the phase error up to $1 \mathrm{mrad}$ are detected, with respect to the error measured at $5 \mathrm{kV}$.

The characterisation at MV voltage is repeated by inserting the divider inside a grounded metallic box with side $80 \mathrm{~cm}$ (Figure 6). The stray capacitive couplings between the high voltage elements and the surrounding metallic surfaces do not significantly modify the divider SF, but lead to an increase of the phase error of $3 \mathrm{mrad}$. A systematic correction for the phase angle can then be considered in case of use of the divider inside the grounded box.

The frequency response is investigated at low voltage

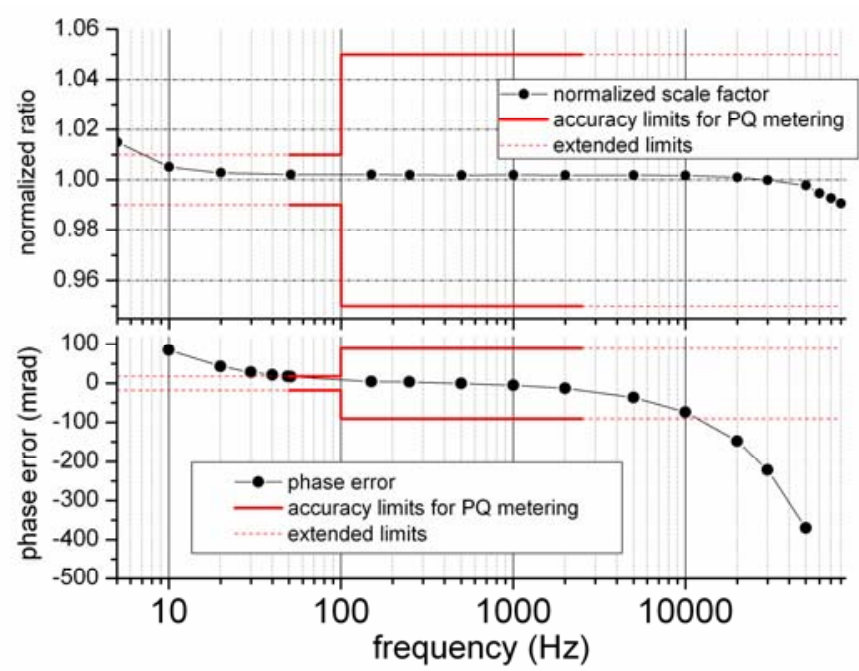

Figure 4. Measured RC plus integrator normalized ratio and phase errors vs frequency.
$(600 \mathrm{~V})$ from $\mathrm{DC}$ up to $30 \mathrm{kHz}$. The divider ratio error complies with the standard requirements up to $1.4 \mathrm{kHz}$. At increasing frequency the divider response amplifies the harmonic amplitude. This effect is exploited to better detect the low amplitude high frequency components. The measured value of the harmonic amplitudes and the THD are then evaluated by introducing the frequency corrections determined in the calibration phase. As an alternative, the correction of the divider frequency behaviour according to the method described in [19], can allow optimisation of its response.

The variation of the $\mathrm{SF}$ in the range $-5^{\circ} \mathrm{C}$ to $40{ }^{\circ} \mathrm{C}$ is measured at low voltage $(600 \mathrm{~V})$. Figure 7 shows the time evolution of the SF variation from the $23^{\circ} \mathrm{C}$ value, with the divider inside a climatic chamber when the chamber temperature is set to $40^{\circ} \mathrm{C}$ and $-5^{\circ} \mathrm{C}$. The SF deviation reached at thermal balance is $+1.7 \%$ and $-1.2 \%$ respectively. The mean temperature coefficient is, thus, 100 $\mathrm{ppm} /{ }^{\circ} \mathrm{C}$. Several hours are needed to reach a steady SF value, due to the amount of mass of the insulated transducer.

\subsection{The measuring instrument}

The transducer voltage and current signals are digitized and PQ parameters are estimated and stored by a NI Compact Reconfigurable I/O (NI Compact RIO) programmable automatic controller platform, which includes a CRIO embedded real-time controller with a 4 slots reconfigurable chassis, equipped with a userprogrammable FPGA.

Two $10 \mathrm{~V}$ A/D cards (16 bit $1 \mathrm{MSample} / \mathrm{s} / \mathrm{ch}$ and 24 bit $50 \mathrm{kSample} / \mathrm{s} / \mathrm{ch}$ ), a GPS synchronisation module and two $\mathrm{SD}$ cards for $\mathrm{PQ}$ parameters recording complete the assembly. The NI Compact RIO platform and the acquisition and elaboration strategy are shown in Figure 8. The quantities of interest: frequency, rms values, harmonics and THDs are evaluated, in real time according to [6] and [7]. In particular, the algorithm for the frequency estimation, over a time interval of $10 \mathrm{~s}$ [6], and the current and voltage rms values are implemented on the FPGA, while the THD and the harmonic content estimation algorithm is implemented in a Real Time Operating

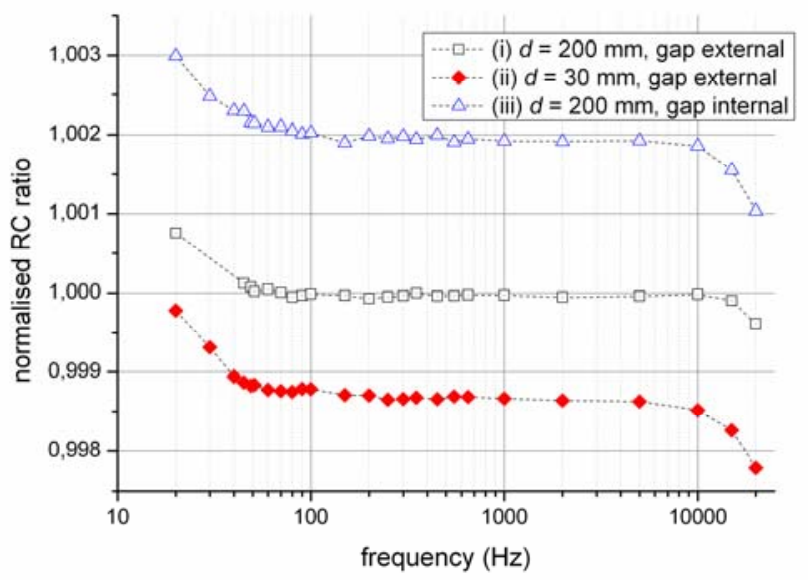

Figure 5. Normalized RC plus integrator ratio for different distances $d$ of the return conductor and coil gap position. 
System. This approach gives a real time estimation of the power quality parameters. Different time aggregation starting from $3 \mathrm{~s}$ can be user selected, as a function of the measurement approach. Presently, four files are stored in the SD cards: one contains the time stamp and the corresponding frequency value, the second stores the time stamp, the voltage and current THD, the rms values of one voltage and three currents and corresponding first harmonics. The last two files contain the time stamp and harmonic contents for one voltage and one current phase, respectively.

\subsection{Uncertainty budget}

The uncertainty budget for the rms current values from $3 \mathrm{~A}$ to $40 \mathrm{~A}$ is shown in Table 2. It is evaluated taking into account the calibration uncertainty of the assigned RC coil ratio and associated measurement instrument (acquisition and evaluation system), its linearity, the effect of the other MV conductors and that of the plant LV conductors. Two estimates are presented: in the first case, a more conservative evaluation is given (i), whilst the second one (ii) is performed considering correction factors and uncertainties relevant to the specific measurement conditions, evaluated taking into account the results obtained in the characterisation carried out as previously detailed. The resulting uncertainty ( $95 \%$ coverage level) reduces from $2 \%$ to $4 \times 10^{-3}$.

As to the rms voltage, and taking into account the contributions respectively due to MV divider linearity from $5 \mathrm{kV}$ to $12 \mathrm{kV}$ and the temperature variation in the range $(23 \pm 10){ }^{\circ} \mathrm{C}$, the measuring system relative uncertainty $(95 \%$ coverage probability) is estimated to be within $2 \times 10^{-3}$.

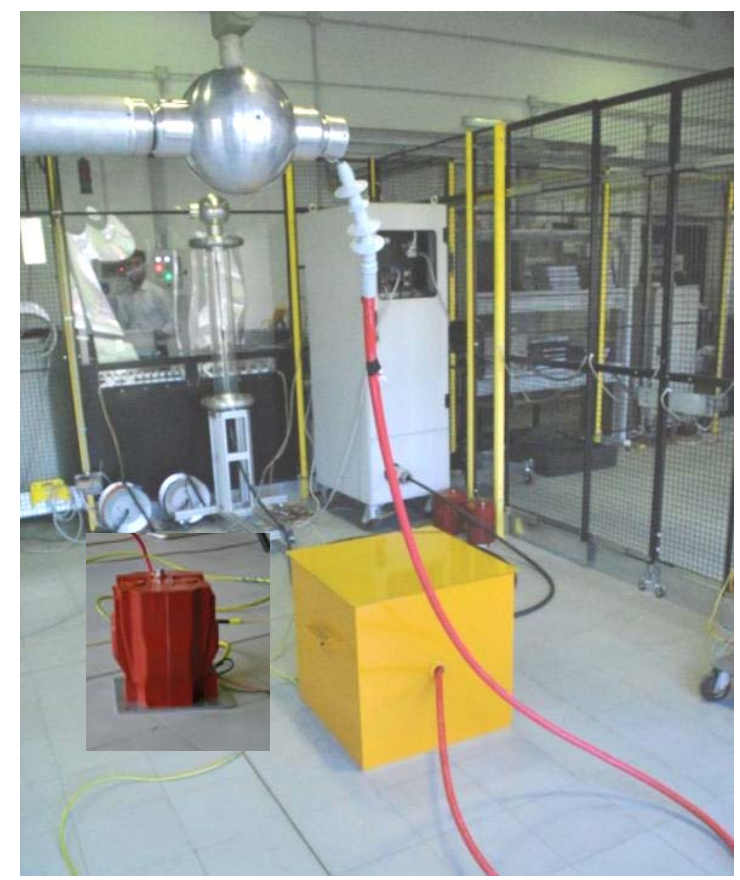

Figure 6. Voltage calibration with the divider inside a metallic grounded box.
Table 2 - Uncertainty budget (rms current from 3 A to 40 A).

\begin{tabular}{|c|c|c|}
\hline Quantity/influence factor & $\begin{array}{l}\text { Relative standard } \\
\text { uncertainty }\left(\cdot 10^{-3}\right) \\
\text { Conf. (i) }\end{array}$ & $\begin{array}{l}\text { Relative standard } \\
\text { uncertainty }\left(\cdot 10^{-3}\right) \\
\text { Conf. (ii) }\end{array}$ \\
\hline $\begin{array}{l}\mathrm{RC} \text { and acquisition system } \\
\text { ratio }\end{array}$ & 0.25 & 0.25 \\
\hline Linearity & 1.5 & 1.0 \\
\hline $\begin{array}{l}\text { Return conductor, gap } \\
\text { position variation and } \\
\text { orthogonality of the } \\
\text { sensor }\end{array}$ & $\begin{array}{l}6.5 \\
30 \mathrm{~mm}<d<350 \mathrm{~mm},\end{array}$ & $\begin{array}{l}0.8 \\
(30 \mathrm{~mm}<d<35 \mathrm{~mm} \text {, } \\
\text { gap external, sensor } \\
\text { centered) }\end{array}$ \\
\hline $\begin{array}{l}\text { Perturbing magnetic field } \\
\text { sources }\end{array}$ & $\begin{array}{l}7 \\
(\mathrm{D}>100 \mathrm{~mm})\end{array}$ & $\begin{array}{l}1.2 \\
(D>600 \mathrm{~mm})\end{array}$ \\
\hline Ambient Temperature & $\begin{array}{l}2.3 \\
T_{a}=(23 \pm 10)^{\circ} \mathrm{C}\end{array}$ & $\begin{array}{l}1.15 \\
\mathrm{~T}_{\mathrm{a}}=(23 \pm 5)^{\circ} \mathrm{C}\end{array}$ \\
\hline Stability and repeatability & 2 & 0.6 \\
\hline $\begin{array}{l}\text { Expanded rel. uncertainty } \\
(95 \% \text { coverage probability) }\end{array}$ & 20 & 4,0 \\
\hline
\end{tabular}

\section{ON-SITE MEASUREMENT}

The developed system has been deployed in a Power Quality measurement campaign carried out in a private $\mathrm{MV} / \mathrm{LV} 15 \mathrm{kV} / 400 \mathrm{~V}, 70 \mathrm{~A}$ private substation. The substation is connected to the public medium voltage distribution network and supplies a factory equipped with two PV generation plants, respectively rated $200 \mathrm{~kW}$ and $800 \mathrm{~kW}$. The private network can absorb up to $800 \mathrm{~kW}$ from the Distribution Network Operator (DNO) and supply up to 1 MVA. Voltage and current are measured on the MV line connecting the $800 \mathrm{kVA}$ step-up transformer to the DNO MV network, by making use of the measurement system equipped with the characterised voltage and current transducers and the measuring system previously described.

In the following, the measurement arrangement, with specific reference to the solutions adopted for connection of the voltage and current transducers is described.

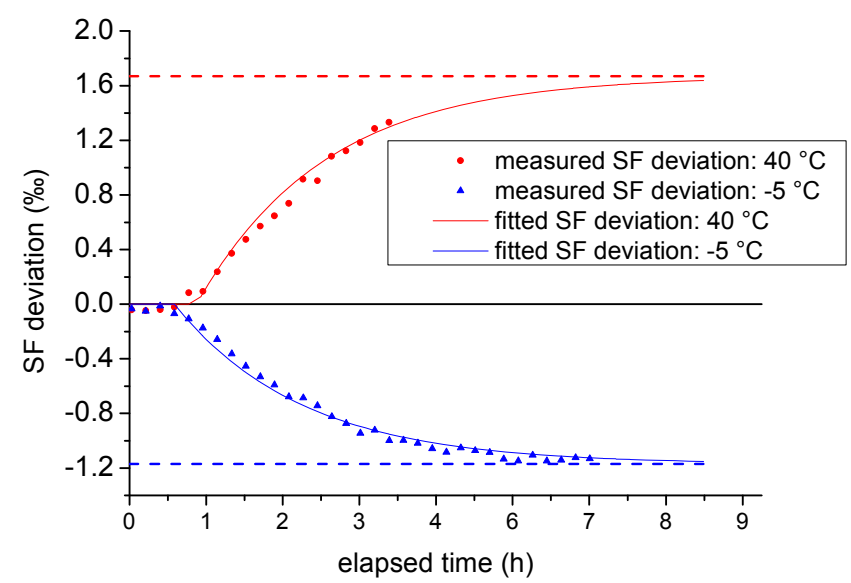

Figure 7. Time evolution of the divider SF relative variation from $23^{\circ} \mathrm{C}$. 


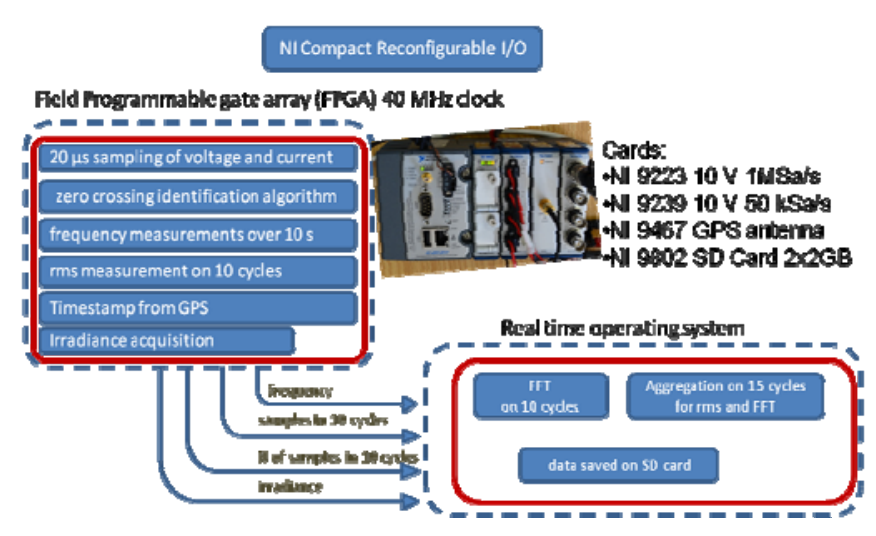

Figure 8. NI Compact RIO and acquisition architecture.

\subsection{The measurement arrangement}

The connection of the sensors to MV level is a crucial point. As regards the voltage sensor, the connection point is made inside the metal enclosed air-insulated switchgear unit on the MV line connecting the $800 \mathrm{~kW}$ step-up transformer to the DNO network. Taking into account the dimensions of the cubicle, the electrical clearances to be respected between the introduced $\mathrm{MV}$ and $\mathrm{LV}$ components and conductors and the surrounding energized, earthed or floating elements, the divider is located outside the unit. To avoid any casual contact with live conductor the divider is placed inside the same grounded metallic box used in the calibration phase (Figure 7) and the MV connection is made by means of a MV cable with suitable terminations. Firm connections to the $\mathrm{MV}$ busbars inside the unit are established by means of bolted connections. The divider

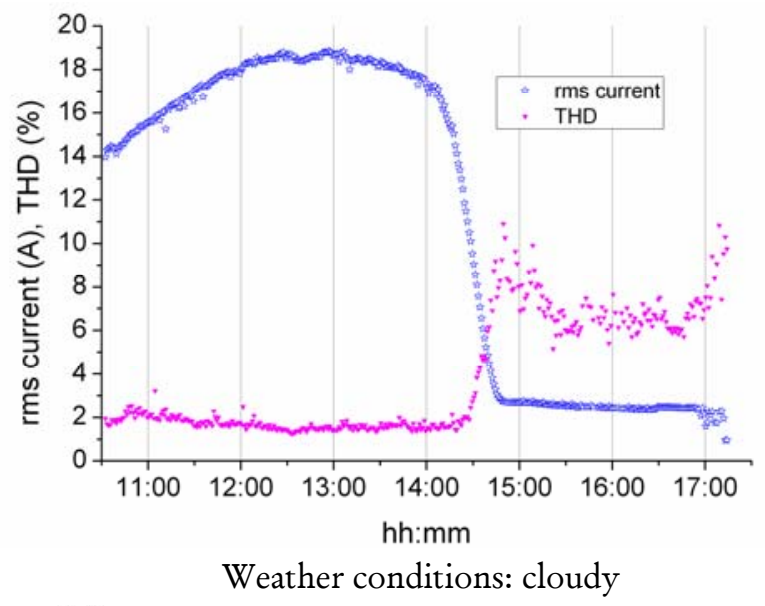

a)

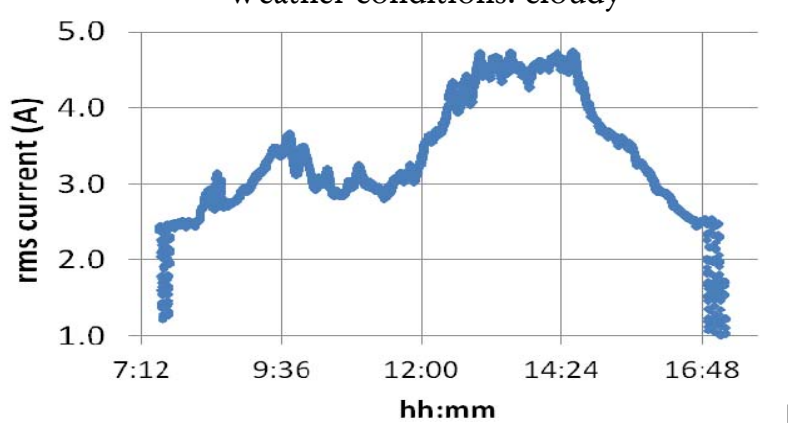

Figure 9. Current measurement on two consecutive days in a late autumn day.

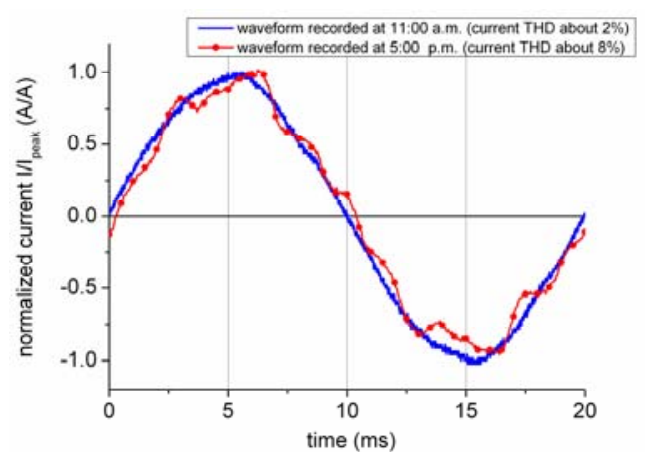

Figure 10. Current waveforms normalized to the peak values detected at 11 a.m., when the current THD is about $2 \%$ a) and at 5 p.m. when THD is around $8 \%$ b).

LO connector is earthed. In order to avoid dangerous overvoltages at the measuring instrument input, a surge arrester is connected between the LV divider output terminal and earth.

Regarding the current sensors, since their positioning on the bare power conductors inside the switchgear unit is not possible, the RCs are centred on the MV insulated cable between the MV transformer of the $800 \mathrm{~kW}$ PV plant and the switchgear unit by a suitable insulating support.

Attention is paid to locate the coil opening gap opposite to the other conductors: keeping it as far as possible from the other MV conductors. Systematic effects of the other phase conductors placed in close proximity were quantified in the laboratory during the coil characterization phase, as described in the previous section.

Measurement of the stray signals due to the currents possibly flowing in the MV cable grounded shields was carried out with the PV plant disconnected. The influence of the stray currents was estimated to be within 5 part per thousand and a few percent for the lower current values. This component is then taken into account in the

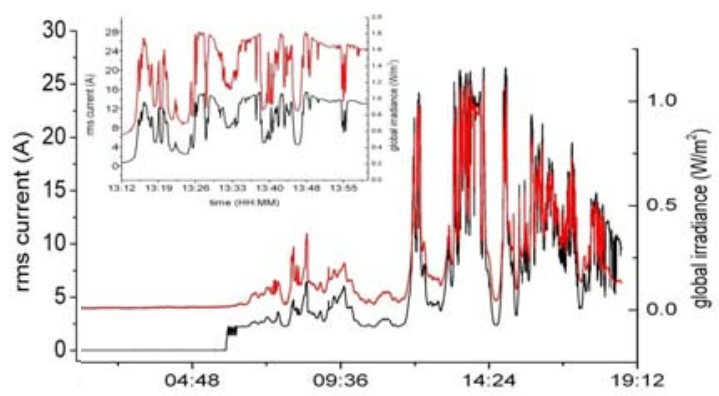

a)

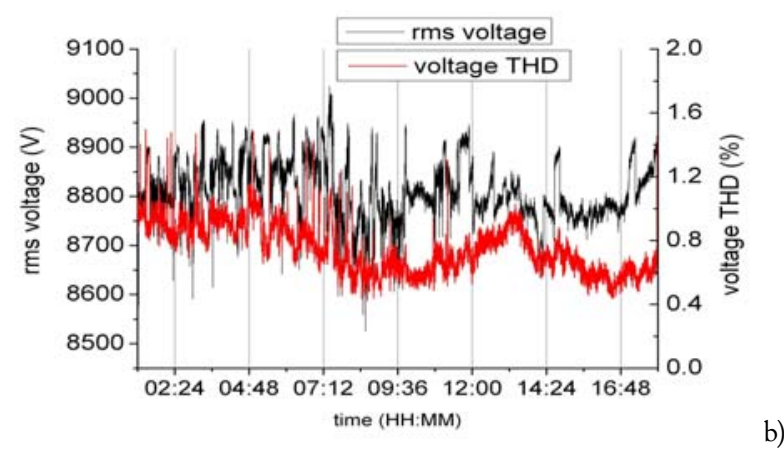

Figure 11. Current, voltage ( $r m s$ and THD) and global irradiance measured on two consecutive days in a late spring day. 

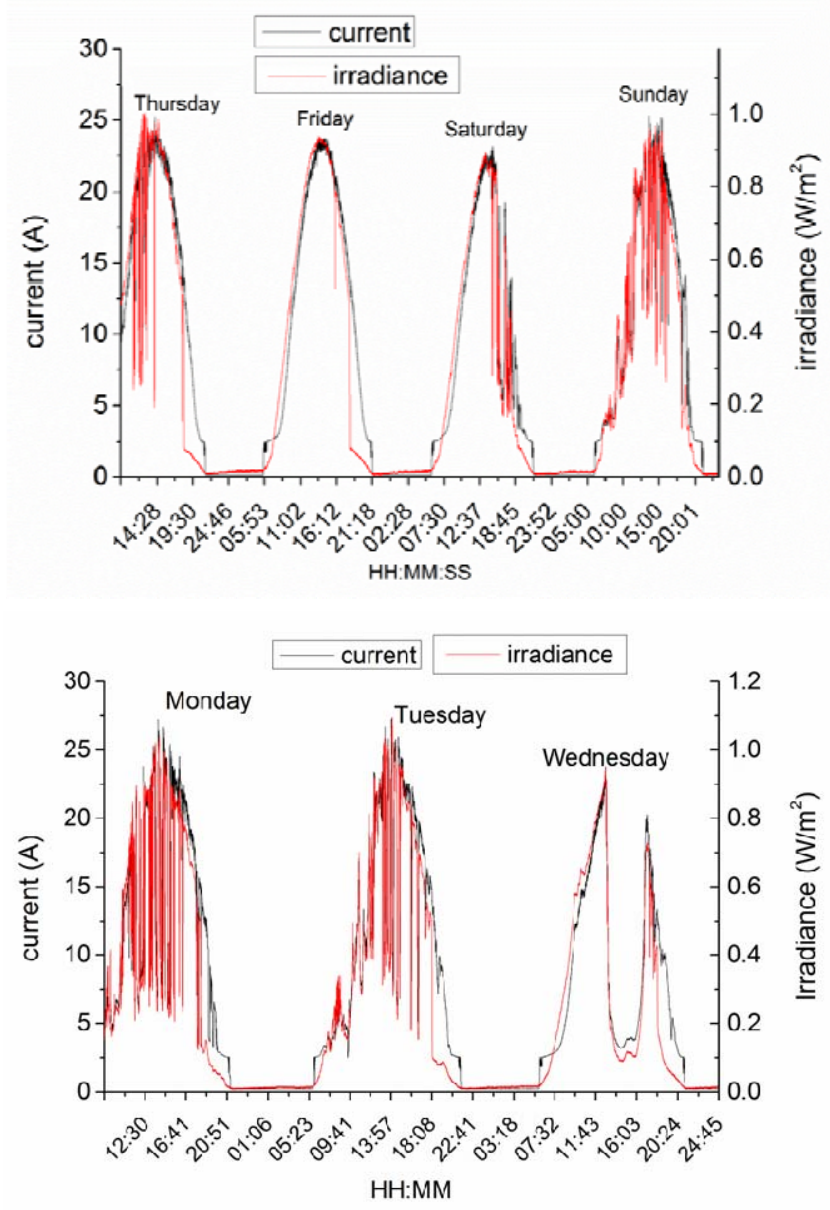

Figure 12. Current and irradiance measurements over one week.

uncertainty budget for the on-site current measurement.

Since the produced current is related to the solar conditions and in particular to the irradiance, a passive pyranometer is installed close to the solar panels [20]. The pyranometer output voltage is digitized by the measurement instrument simultaneously to the acquired current signal.

\section{RESULTS AND DISCUSSION}

Voltage and current measurement are performed under different weather conditions during different seasons.

The current and voltage signals are digitised with $50 \mathrm{kHz}$ sampling frequency and the frequency, rms, THD, harmonic parameters are evaluated. A preliminary current analysis, carried out on the current waveforms with higher sampling frequencies (up to $500 \mathrm{kHz}$ ), does not detect any harmonic of the $18 \mathrm{kHz}$ fundamental one.

A quite significant variation in the maximum $\mathrm{rms}$ currents is found depending on the weather conditions, as shown in Figure 9. To give evidence on the waveform distortion which dramatically depends on the generated current amplitude, thus on the weather conditions, Figure 10 is introduced. It shows one period of the injected currents, normalized to their peak values, recorded at 11:00 a.m. during a sunny day when the related THD is about $2 \%$ and the rms is about $16 \mathrm{~A}$ and at 5 p.m. when the THD

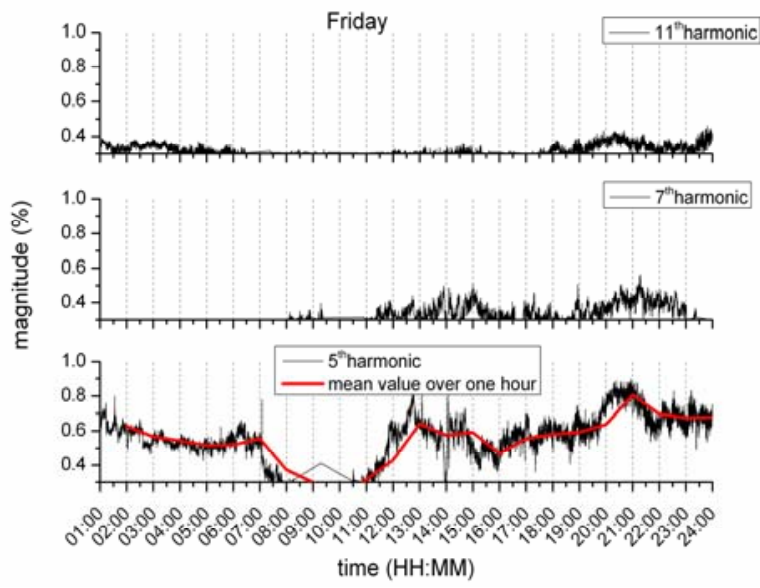

Figure 13. Daily behavior of 5th, 7th and 11th voltage harmonics detected on a working day (amplitude normalized to the $50 \mathrm{~Hz}$ component).

reaches $8-10 \%$ while the rms falls down to $2 \mathrm{~A}$ (see Figure 9).

The measured rms current and irradiance show quite the same behaviour (Figure 11a). As expected, the current THD significantly increases for values lower than some amperes. When the rms current decreases from 8 A (measured irradiance $0.3 \mathrm{~W} / \mathrm{m}^{2}$ ) to $3 \mathrm{~A}$ (measured irradiance $0.2 \mathrm{~W}$ $\left./ \mathrm{m}^{2}\right)$, the corresponding current THD increases from $2.4 \%$ to $8.5 \%$. However, the corresponding measured voltage behaviour remains quite unchanged $(\mathrm{THD}=0.6 \% / 0.7 \%$ ). This can be explained considering the low value of the ratio of the PV plant power to the short-circuit power at the point of common coupling.

The simultaneously measured voltage is shown in Figure $11 \mathrm{~b}$. The rms voltage values slightly increase during the weather conditions: sunny evening/night hours.

The harmonic analysis detects the presence of two significant components (the 5 th and the 7 th).

Analysis of currents recorded during one week period shows, as expected, a marked dependence on the weather conditions (Fig 12).

As regards the voltage, the measured behaviour again indicates an increase of the voltage during the nights. Maximum variations of the rms voltage are found within $\pm 3 \%$. The slight voltage increase (about $1 \%$ ) during the night is more evident if the mean rms voltages measured in five working days in the day-time hours (7-19) are compared with those registered during the night (19-7). On the contrary, this voltage increase is far less evident or nor detected during the weekends.

An example of the performances of the measuring system as regards the voltage harmonic analysis is shown in Figure 13, where the daily behaviour of the three most significant harmonics, normalised to the fundamental one, are shown. Harmonics voltage variations down to some hundreds of ppm of the fundamental one are well evidenced by the system. The uncertainty associated with the harmonic measurement is estimated to be within $0.25 \%$ of the power frequency component. 


\section{CONCLUSIONS}

PQ measurement accuracy in MV and HV plants can significantly depend on the performances of the used current and voltage transducers in relation to the actual measurement conditions. The measurement uncertainty can be estimated and reduced by carrying out a careful characterisation of the used transducers. Characterisation procedures under conditions as close as possible to the onsite ones have been experimented on a current and voltage measurement system which includes a clamp-on and flexible RC current sensor, whose expected on-site accuracy is of the order of some percent, and an accuracy class 0.5 voltage divider. By taking into account the results obtained in the characterisation phase the estimated uncertainty of the current and voltage measurements carried out on-site by the developed systems are reduced to 6 and 2 part per thousand, respectively.

\section{ACKNOWLEDGEMENT}

The research leading to the results here described is part of the European Metrology Research Program (EMRP), which is jointly funded by the EMRP participating countries within EURAMET and the European union. Moreover, the authors acknowledge the FTM (Fabbrica Trasformatori di Misura) factory for its valuable contribution in the development of the voltage divider and COS.PEL which made the MV station of the Mondovi (Italy) plant available for the PQ measurements.

\section{REFERENCES}

[1] EN 50160, Voltage characteristics of electricity supplied by public distribution networks, 2011-05.

[2] P. Kadurek, J. F. G. Cobben , and W. L. Kling "Smart MV/LV transformer for future grids”, SPEEDAM 2010, International Symposium on Power Electronics, Electrical Drives, Automation and Motion.

[3] N. R. Browne, T. J. Browne, S. Elphick, "Monitoring Intelligent Distribution Power Systems - A Power Quality Plan”, Harmonics and Quality of Power (ICHQP), $201014^{\text {th }}$ International Conference on, pp.1-7.

[4] S. Elphick, V. Smith, V. Gosbell, R. Barr, "Australian Long Term Power Quality Survey Project Update”.

[5] Mindykowski, "Fundamentals of electrical power quality assessment” XVII IMEKO World Congress, 22-27 June 2003 Dubrovnik, Croatia, pp. 552-557.

[6] IEC 61000 "EMC Part 4-30: Power quality measurement methods", 2009.

[7] IEC 61000 "EMC Part 4-7: "General guide on harmonics and interharmonics measurements and instrumentation for power supply systems and equipment connected thereto", 2009.

[8] IEC/TR 61869-103 "Instrument transformers - The use of instrument transformers for Power Quality measurements", 2012.

[9] A. Cataliotti, D. Di Cara, E. Emanuel, S. Nuccio., "Current Transformers Effects on the Measurement of Harmonic Active Power in LV and MV Networks", IEEE Trans. on PD, vol. 26, no. 1, pp. 360-368, 2011.

[10] D. Gallo, C. Landi, M. Luiso, E. Fiorucci, G. Bucci, F. Ciancetta, "Realization and Characterization of an Electronic Instrument Transducer for MV Networks with Fiber Optic Insulation", WSEAS TRANSACTIONS ON POWER SYSTEMS, Issue 1, Volume 8, January 2013.

[11] N. Locci, C. Muscas, S. Sulis, "Experimental comparison of MV voltage transducers for power quality applications", I2MTC 2009 - International Instrumentation and Measurement Technology Conference, Singapore, 5-7 May 2009.

[12] M. Faifer, S, Toscani, R. Ottoboni, "Electronic Combined Transformer for Power-Quality Measurements in HighVoltage Systems", IEEE Trans. on Instrum. and Meas., vol. 60, no. 6, June 2011.

[13] S. Kucuksari, G. G. Karady "Experimental Comparison of Conventional and Optical Current Transformers, IEEE Trans. On Power Delivery, vol. 25, no. 4, October 2010, pp. 2455-2463.

[14] Agilent technologies 3458A multimeters User's guide, http://cp.literature.agilent.com/litweb/pdf/03458-90014.pdf

[15] IEC 60044-8 - Instrument transformers Part 8: Electronic current transformers, 2004.

[16] Delle Femine, A.; Gallo, D.; Landi, C.; Luiso, M., "PowerQuality Monitoring Instrument With FPGA Transducer Compensation," Instrumentation and Measurement, IEEE Transactions on , vol.58, no.9, pp.3149,3158, Sept. 2009.

[17] J.D. Ramboz, "Machinable Rogowski Coils, Design and Calibraton”, IEEE Trans. on Instrum. and Meas., vol. 45, no. 6, April 1996.

[18] M. Chiampi, G. Crotti, A. Morando "Evaluation of Flexible Rogowski Coil Performances in Power Frequency Applications", IEEE Trans. on IM, vol. 60, no. 3, pp. 854-862, 2011.

[19] G. Crotti, D. Gallo, D. Giordano, C. Landi, M. Luiso “A real-time compensation method for $\mathrm{MV}$ voltage transducer for power quality analysis”, Proc. of 19th IMEKO TC 4 Symposium and $17^{\text {th }}$ IWADC Workshop Advances in Instrumentation and Sensors Interoperability, July 18-19, 2013, Barcelona, Spain.

[20] M Patsalides, D. Evagorou, G. Makrides, Z. Achillides, G. E. Georghiou, A. Stavrou, V. Efthimiou, B. W. Schmitt, J. H. Werner "The Effect of Solar Irradiance on the Power Quality Behaviour of Grid Connected Photovoltaic Systems". 